\title{
Dynamical effects of a one-dimensional multibarrier potential of finite range
}

\author{
D.Bar $^{a}$ and L.P.Horwitz ${ }^{a, b}$ \\ ${ }^{a}$ Department of Physics, Bar Ilan University, Ramat Gan, Israel \\ ${ }^{b}$ Raymond and Beverly Sackler Faculty of Exact Science, School of Physics, Tel Aviv \\ University, Ramat Aviv, Israel
}

\begin{abstract}
We discuss the properties of a large number $N$ of one-dimensional (bounded) locally periodic potential barriers in a finite interval. We show that the transmission coefficient, the scattering cross section $\sigma$, and the resonances of $\sigma$ depend sensitively upon the ratio of the total spacing to the total barrier width. We also show that a time dependent wave packet passing through the system of potential barriers rapidly spreads and deforms, a criterion suggested by $\mathrm{Za}$ slavsky for chaotic behaviour. Computing the spectrum by imposing (large) periodic boundary conditions we find a Wigner type distribution. We investigate also the $S$-matrix poles; many resonances occur for certain values of the relative spacing between the barriers in the potential.
\end{abstract}

PACS number(s): 03.65.Nk, 02.10.Yn, 05.45.Pq 


\section{INTRODUCTION}

Quantum systems with chaotic-like properties in the presence of tunneling through a single barrier have been studied recently [1]. The effect was attributed to the complexity of the wave function and its time dependence in the neighbourhood of the barrier, where Zaslavsky's criterion for the decrease of the Ehrenfest time [2] is expected to be satisfied. We discuss here several aspects of scattering from a one-dimensional locally periodic potential barrier system $[3,4]$ which is composed of a large number $N$ of identical potential barriers densely arrayed along a finite section of the $x$ axis. We discuss both cases of finite and infinite $N$. Parameters such as the transmission coefficient, the scattering cross section and the energy spectrum depend sensitively upon the ratio of the total spacing between the potential barriers to their total width. For example, it is shown in Section 2 for finite $N$ and for both cases of $e>v$ and $v>e$ and in Sections 3, 4 and 5 for infinite $N$ that as this ratio grows the transmission coefficient tends rapidly to unity. An approaching particle can, therefore, be transmitted unattenuated in its amplitude through these barriers without having to increase its energy, even when $v \gg>$ (for the $v>e$ case). Also, it has been shown, using the level statistics [5] of the energy spectrum of this dense system, that when this ratio increases the dense system appears to become chaotic-like in the sense of ref [1]. These chaotic-like characteristics emerge also, as will be seen in Section 4, when we study the passage of a Gaussian wave packet through the dense system. We show also that for both cases $e>v$ and $v>e$ the resonances of the scattering cross section depend strongly upon this ratio.

Frishman and Gurvitz [6] have pointed out that the multiple barrier structure is important to study. They analyse the finite multiple well problem using a tight binding approximation, and find a miniband structure (with some similarity to the Kronig-Penney spectrum) which may correspond to the property of rapid approach to complete transmission that we find from certain values of the ratio of spacing to barrier width denoted in the following by $c$. The exact solution that we study permits us to investigate the very sensitive dependence of the transmission phenomena, as well as the distortion of wave packets with high precision for both small and large number $\mathrm{N}$ of barriers restricted to a fixed total interval. We find a simple form for the limit $N \rightarrow \infty$, which retains the very sensitive dependence on $c$.

The configuration of a large density of one-dimensional barriers in a small range can occur in physical systems with planar defects such as successive evaporated layers or traps $[7,8]$; higher dimension analogs may also be realized.

In section 2 we use a numerical model of this system where the number $N$ of potential barriers is finite. We discuss both cases of $e>v$ and $v>e$. In Sections 3, 4 and 5 we discuss, using the transfer matrix method, the limit of an infinite number of potential barriers densely arrayed along a finite section of the $x$ axis. The $e>v$ case is discussed in Sections 3-4, and the $v>e$ case in Section 5. In both cases we discuss the transmission probability, the scattering cross section, the poles of this cross section and the energy spectrum. We discuss also the level statistics of the energy spectrum of this dense system, and the properties of a time dependent wave packet that passes through it.

We remark that although we study a system of $N$ barriers that is locally periodic, all of these barriers are contained, even for $N \rightarrow \infty$, in a finite interval. The system is therefore

not equivalent to a Kronig-Penney type model [3]; the emergence of the band like structure 
as seen in Figure 3 appears to be due to the local (internal) periodicity but does not follow from global crystal translation symmetry, this periodicity is, moreover, not in the particle momentum, but in the total potential width.

We believe that similar results may also be obtained for other similar systems like, for example, the $N$ one dimensional periodic potential wells in a finite interval.

\section{THE $4 N \times 4 N$ MATRIX APPROACH FOR THE ENSEMBLE OF POTENTIAL BARRIERS}

The array of $N$ potential barriers discussed here is located along a finite section of the positive $x$ axis beginning with the point $x=0$. We denote the overall width of all the potential barriers by $a$, and the total width of all the interim spaces separating them by $b$. That is, in an array of $N$ potential barriers the width of each one is $\frac{a}{N}$, and since in such an array there are $(N-1)$ separating spaces, the width of each one is $\frac{b}{(N-1)}$. A sketch of our array is shown in figure 1. To this array approaches from the negative half of the $x$ axis a plane wave $e^{i k x}$, where $k=\left(\frac{2 m e}{\hbar^{2}}\right)^{\frac{1}{2}}$.

We consider both cases: $e>v$, and $v>e$, where $v$ is the constant height of each potential barrier, and $e$ is the energy of the coming wave function. We begin with the $e>v$ case and write the following set of $4 N$ simultaneous linear equations obtained from the boundary conditions at the left and right hand sides of all the $N$ potential barriers [3,4] (see figure 1).

$$
\begin{aligned}
& 1+A=B+C \\
& i k-i k A=i q B-i q C \\
& B e^{i \frac{q a}{N}}+C e^{-i \frac{q a}{N}}=D e^{\frac{i k a}{N}}+E e^{-\frac{i k a}{N}} \\
& i q B e^{i \frac{q a}{N}}-i q C e^{-i \frac{q a}{N}}=i k D e^{\frac{i k a}{N}}-i k E e^{-\frac{i k a}{N}} \\
& D e^{i k\left(\frac{a}{N}+\frac{b}{(N-1)}\right)}+E e^{-i k\left(\frac{a}{N}+\frac{b}{(N-1)}\right)}=F e^{i q\left(\frac{a}{N}+\frac{b}{(N-1)}\right)}+G e^{-i q\left(\frac{a}{N}+\frac{b}{(N-1)}\right)} \\
& i k D e^{i k\left(\frac{a}{N}+\frac{b}{(N-1)}\right)}-i k E e^{-i k\left(\frac{a}{N}+\frac{b}{(N-1)}\right)}=i q F e^{i q\left(\frac{a}{N}+\frac{b}{(N-1)}\right)}-i q G e^{-i q\left(\frac{a}{N}+\frac{b}{(N-1)}\right)} \\
& \text { (n. } \\
& R e^{i k\left(\frac{a(N-1)}{N}+b\right)}+S e^{-i k\left(\frac{a(N-1)}{N}+b\right)}=T e^{i q\left(\frac{a(N-1)}{N}+b\right)}+U e^{-i q\left(\frac{a(N-1)}{N}+b\right)} \\
& i k R e^{i k\left(\frac{a(N-1)}{N}+b\right)}-i k S e^{-i k\left(\frac{a(N-1)}{N}+b\right)}=i q T e^{i q\left(\frac{a(N-1)}{N}+b\right)}-i q U e^{-i q\left(\frac{a(N-1)}{N}+b\right)} \\
& T e^{i q(a+b)}+U e^{-i q(a+b)}=Z e^{i k(a+b)} \\
& i q T e^{i q(a+b)}-i q U e^{-i q(a+b)}=i k Z e^{i k(a+b)},
\end{aligned}
$$

where $q=\sqrt{\frac{2 m(e-v)}{\hbar^{2}}}$. The former set can be written in a matrix form as $T x=\zeta$, where $T$ is the square matrix with $4 N$ rows and $4 N$ columnns whose elements are given in the set (1). We denote by $x$ the unknown vector with the $4 N$ unknowns $(A, B, C \ldots . Z)$, and $\zeta$ is the constant vector whose two first elements are -1 and $-i k$, and all its other elements are zero. As can be seen from the set (1) all the $4 N$ unknowns are obtained after dividing by the coefficient of the incoming wave so that $|A|^{2}$ and $|Z|^{2}$ (see the first two and the last two equations of the set (1)) are the coefficients of reflection and transmission respectively. In 
order to calculate the value of $|Z|^{2}$ we have to solve the $4 N$ simultaneous linear equations $T x=\zeta$. This can be done, especially for large $N$, only by numerical methods. We denote the constant length $a+b$ of the $x$ axis along which the system is arrayed by $L$, and define $b=a c$. Thus, we can express $a$ and $b$ in terms of $L$ and $c$ as follows

$$
a=\frac{L}{1+c} \quad b=\frac{L c}{1+c}
$$

The continuous curve in figure 2 shows the transmission coefficient $|Z|^{2}$ as a function of $c$ in the range $1 \leq c \leq 35$ for $N=30$, and the dashed curve is for $N=40$. The other parameters are assigned the following values: $v=100, e=200, \hbar=1, m=\frac{1}{2}, L=30$. It is seen that the transmission coefficients, for both values of $N$, tend to unity when $c$ grows, but for the larger $N$, smaller values of $c$ suffice for the transmission coefficient to approach unity. That is, when the number of barriers increases, the approaching wave function passes unattenuated in its amplitude through these barriers even for relatively small values of $c$. We note that we obtain the same result of a unity value for the transmission coefficient also when the restriction to a constant total length of the system is relaxed, as seen from figure 3. The dashed curve in figure 3 shows the transmission coefficient as a function of the total width $a$ when $N=60$, and the continuous curve is for $N=120$. For both curves we have assigned to $b$ the value $b=\frac{a}{2}$. The potential $v$ is 100 and the energy $e$ is 200 as for figure 2. From the dashed graph, for $N=60$, we see that the transmission coefficient has an almost constant periodic pattern repeated as a function of $a$. In each one of these patterns the transmission coefficient oscillates near the value of 1, except at the beginning and end of each of these patterns where it drops to zero. In the continuous curve, for $N=120$, as in the dashed one, we have also a similar pattern repeated over the $a$ axis, but this time the width of each such pattern is almost double. Checking the pattern of the dashed curve we see that the first drop of the transmission coefficient to zero occurs at $a \approx 9$, whereas the corresponding drop in the continuous curve is at $a \approx 20$. For $N=240$ (this graph is not shown here) the transmission coefficient remains in the immediate neighbourhood of 1 when $1 \leq a \leq 40$. At about $a=40$ this coefficient drops to 0 , and then at $a=47$ it rises to 1 and remains in the neighbourhood of 1 until $a \approx 87$. Thus, as $N$ becomes larger the transmission coefficient appears to remain in the neighbourhood of unity for larger intervals of $a$, so that as $N \rightarrow \infty$ these intervals would appear to become infinite in extent. In this limit one finds agreement with the closed form we obtain in Eq (15).

We, now, discuss the case $v>e$. In this case the set (1) has to be changed to take into account the tunneling intervals, that is,

$$
\begin{aligned}
1+A & =B+C \\
i k-i k A & =-q B+q C \\
B e^{-\frac{q a}{N}}+C e^{\frac{q a}{N}} & =D e^{\frac{i k a}{N}}+E e^{-\frac{i k a}{N}} \\
-q B e^{-\frac{q a}{N}}+q C e^{\frac{q a}{N}} & =i k D e^{\frac{i k a}{N}}-i k E e^{-\frac{i k a}{N}} \\
D e^{i k\left(\frac{a}{N}+\frac{b}{(N-1)}\right)}+E e^{-i k\left(\frac{a}{N}+\frac{b}{(N-1)}\right)} & =F e^{-q\left(\frac{a}{N}+\frac{b}{(N-1)}\right)}+G e^{q\left(\frac{a}{N}+\frac{b}{(N-1)}\right)} \\
i k D e^{i k\left(\frac{a}{N}+\frac{b}{(N-1)}\right)}-i k E e^{-i k\left(\frac{a}{N}+\frac{b}{(N-1)}\right)} & =-q F e^{-q\left(\frac{a}{N}+\frac{b}{(N-1)}\right)}+q G e^{q\left(\frac{a}{N}+\frac{b}{(N-1)}\right)}
\end{aligned}
$$




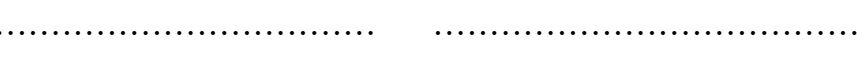

$$
\begin{aligned}
& R e^{i k\left(\frac{a(N-1)}{N}+b\right)}+S e^{-i k\left(\frac{a(N-1)}{N}+b\right)}=T e^{-q\left(\frac{a(N-1)}{N}+b\right)}+U e^{q\left(\frac{a(N-1)}{N}+b\right)} \\
& i k R e^{i k\left(\frac{a(N-1)}{N}+b\right)}-i k S e^{-i k\left(\frac{a(n-1)}{n}+b\right)}=-q T e^{-q\left(\frac{a(N-1)}{N}+b\right)}+q U e^{q\left(\frac{a(n-1)}{n}+b\right)} \\
& T e^{-q(a+b)}+U e^{q(a+b)}=Z e^{i k(a+b)} \\
& -q T e^{-q(a+b)}+q U e^{q(a+b)}=i k Z e^{i k(a+b)}
\end{aligned}
$$

In the set (3) $k$ is the same as the $k$ of the set (1) whereas $q$ is $q=\left(\frac{2 m(v-e)}{\hbar^{2}}\right)^{\frac{1}{2}}$. In $(3)$ the wave functions inside the potential barriers contain real exponentials. This changes the former sinusoidal character of these wave functions (see the set (1)) to a hyperbolic one. The continuous curve in figure 4 shows the graph of the transmission coefficient $|Z|^{2}$ as a function of $c$ for $N=30$, and the dashed curve is for $N=50$. The potential $v$ is 200 , and the energy $e=180$. The total length of the system and the range of $c$ are the same as in figure 2, that is, $L=30$, and $1 \leq c \leq 35$. We see from both graphs that although $v>e$ the transmission coefficient tends to unity as $c$ grows, but this approach to unity is faster and for smaller values of $c$, when $N$ is larger. The same result is obtained if the condition of a constant total length of the system is relaxed as shown in figure 5 , which shows the transmission coefficient as a function of the number $N$ of potential barriers of the system. In this figure we take the total width $a$ of all the potential barriers to be 8 , and the total interval $b$ to be $\frac{a}{2}\left(c=\frac{1}{2}\right)$. The energy and the potential are assigned the values of 200 and 202 respectively. In this figure we see that the transmission coefficient has oscillating type behaviour when the number of barriers is small. At the larger values of $N$ the transmission coefficient is in the close neighbourhood of unity.

In summary, we see from this $4 N \times 4 N$ matrix method applied to both cases of $e>v$ and $v>e$, and for either a constant or variable total length of the system, that when the ratio $c$ increases the transmission coefficient tends to 1 , and when the number of potential barriers grows it tends to unity already at small values of $c$.

\section{THE TRANSFER MATRIX METHOD FOR THE $E>V$ CASE}

We discuss, now, the multiple barrier system by the transfer matrix method $[3,4,11]$, and in order to exploit its symmetry the dense array is assumed to be arranged between the points $x=-\frac{a+b}{2}$ and $x=\frac{a+b}{2}$, where $a$ and $b$ has the same meaning as in the former section. We discuss first the $e>v$ case. Using the terminology of Merzbacher [3] we can write the following transfer matrix equation which governs the behaviour of the bounded potential system.

$$
\left[\begin{array}{l}
A_{2 n+1} \\
B_{2 n+1}
\end{array}\right]=P^{(n)} P^{(n-1)} \ldots P^{(2)} P^{(1)}\left[\begin{array}{c}
A_{0} \\
B_{0}
\end{array}\right]
$$

where $A_{2 n+1}$ and $B_{2 n+1}$ are the amplitudes of the transmitted and reflected parts respectively of the wave function from the $n$th potential barrier. $A_{0}$ is the coefficient of the initial wave that approaches the potential barrier system, and $B_{0}$ is the coefficient of the reflected wave from the first barrier. $P^{(n)}$ is the product of three two dimensional matrices 


$$
\begin{aligned}
& P^{(n)}=M_{n} T \grave{M}_{n}= \\
& =\left[\begin{array}{cc}
e^{-i k\left(\frac{(n-1) b}{N-1}+\frac{(2 n-1) a}{2 N}\right)} & 0 \\
0 & e^{i k\left(\frac{(n-1) b}{N-1}+\frac{(2 n-1) a}{2 N}\right)}
\end{array}\right]\left[\begin{array}{ll}
T_{11} & T_{12} \\
T_{21} & T_{22}
\end{array}\right]\left[\begin{array}{cc}
e^{i k\left(\frac{(n-1) b}{N-1}+\frac{(2 n-3) a}{2 N}\right)} & 0 \\
0 & e^{-i k\left(\frac{(n-1) b}{N-1}+\frac{(2 n-3) a}{2 N}\right)}
\end{array}\right]
\end{aligned}
$$

The middle matrix $T$ does not depend on $n[12]$ and its components are given by

$$
\begin{aligned}
& T_{11}=\cos \left(\frac{a q}{N}\right)+i \frac{\xi}{2} \sin \left(\frac{a q}{N}\right), \quad T_{12}=i \frac{\eta}{2} \sin \left(\frac{a q}{N}\right) \\
& T_{21}=-i \frac{\eta}{2} \sin \left(\frac{a q}{N}\right), \quad T_{22}=\cos \left(\frac{a q}{N}\right)-i \frac{\xi}{2} \sin \left(\frac{a q}{N}\right)
\end{aligned}
$$

Here $k$ is $\sqrt{\frac{2 m e}{\hbar^{2}}}, q$ is $\sqrt{\frac{2 m(e-v)}{\hbar^{2}}}$, and $\xi$ and $\eta$ are given by

$$
\xi=\frac{q}{k}+\frac{k}{q}, \quad \eta=\frac{q}{k}-\frac{k}{q}
$$

As can be seen from Eq (5) the product of each neighbouring diagonal matrices $\grave{M}_{n} M_{n-1}$ is constant for each $n$. That is, $\grave{M}_{n} M_{n-1}=\left[\begin{array}{cc}e^{\frac{i k b}{N-1}} & 0 \\ 0 & e^{-\frac{i k b}{N-1}}\end{array}\right]$. Thus, we may write Equation (4) as

$$
\begin{aligned}
& {\left[\begin{array}{l}
A_{2 n+1} \\
B_{2 n+1}
\end{array}\right]=\left[\begin{array}{cc}
e^{-i k\left(a+b-\frac{a}{2 N}\right)} & 0 \\
0 & e^{i k\left(a+b-\frac{a}{2 N}\right)}
\end{array}\right]\left[\begin{array}{cc}
T_{11} & T_{12} \\
T_{21} & T_{22}
\end{array}\right]\left(\left[\begin{array}{cc}
e^{\frac{i k b}{N-1}} & 0 \\
0 & e^{-\frac{i k b}{N-1}}
\end{array}\right]\left[\begin{array}{cc}
T_{11} & T_{12} \\
T_{21} & T_{22}
\end{array}\right]\right)^{n-1} .} \\
& \cdot\left[\begin{array}{cc}
e^{\frac{-i k a}{2 N}} & 0 \\
0 & e^{\frac{i k a}{2 N}}
\end{array}\right]\left[\begin{array}{c}
A_{0} \\
B_{0}
\end{array}\right]
\end{aligned}
$$

If we take the limit of a very large $N$, we obtain for the right hand side of the potential barrier system at the point $x=\frac{a+b}{2}$ where $n=N$

$$
\left[\begin{array}{l}
A_{2 N+1} \\
B_{2 N+1}
\end{array}\right]=\left[\begin{array}{cc}
e^{-i k(a+b)} & 0 \\
0 & e^{i k(a+b)}
\end{array}\right]\left(\left[\begin{array}{cc}
e^{\frac{i k b}{N}} & 0 \\
0 & e^{-\frac{i k b}{N}}
\end{array}\right]\left[\begin{array}{cc}
T_{11} & T_{12} \\
T_{21} & T_{22}
\end{array}\right]\right)^{N}\left[\begin{array}{l}
A_{0} \\
B_{0}
\end{array}\right]
$$

We note that the last equation can be discussed from the eigenvalue point of view [3,4]. That is, by finding the appropriate eigenvalues from the suitable characteristic equation (a similar method has been applied to the finite $N$ potential barrier system in [4]). In the following we adopt a more analytical and exact approach that yields the same results obtained from the former numerically-oriented method.

The expression under the exponent $N$ in Eq (9) can be written, using the set (6), in the limit of very large $N$, as

$$
\begin{aligned}
& \left(\left[\begin{array}{cc}
e^{\frac{i k b}{N}} & 0 \\
0 & e^{-\frac{i k b}{N}}
\end{array}\right]\left[\begin{array}{cc}
\cos \left(\frac{a q}{N}\right)+i \frac{\xi}{2} \sin \left(\frac{a q}{N}\right) & i \frac{\eta}{2} \sin \left(\frac{a q}{N}\right) \\
-i \frac{\eta}{2} \sin \left(\frac{a q}{N}\right) & \cos \left(\frac{a q}{N}\right)-i \frac{\xi}{2} \sin \left(\frac{a q}{N}\right)
\end{array}\right]\right)^{N} \cong \\
& \cong\left(\left[\begin{array}{cc}
\left(1+\frac{i k b}{N}\right)\left(1+\frac{i \xi}{2}\left(\frac{a q}{N}\right)\right) & i\left(1+\frac{i k b}{N}\right) \frac{\eta}{2}\left(\frac{a q}{N}\right) \\
-i\left(1-\frac{i k b}{N}\right) \frac{\eta}{2}\left(\frac{a q}{N}\right) & \left(1-\frac{i k b}{N}\right)\left(1-\frac{i \xi}{2}\left(\frac{a q}{N}\right)\right)
\end{array}\right]\right)^{N} \cong \\
& \cong\left(1+\frac{i}{N}\left(\left[\begin{array}{cc}
k b+a q \frac{\xi}{2} & a q \frac{\eta}{2} \\
-a q \frac{\eta}{2} & -\left(k b+a q \frac{\xi}{2}\right)
\end{array}\right]\right)\right)^{N} \cong\left(1+\frac{i}{N}\left(\left(k b+a q \frac{\xi}{2}\right) \sigma_{3}+i a q \frac{\eta}{2} \sigma_{2}\right)\right)^{N},
\end{aligned}
$$


where $\sigma_{2}$ and $\sigma_{3}$ are the standard Pauli matrices $\sigma_{2}=\left[\begin{array}{cc}0 & -i \\ i & 0\end{array}\right], \sigma_{3}=\left[\begin{array}{cc}1 & 0 \\ 0 & -1\end{array}\right]$. Using the relation $\lim _{n \rightarrow \infty}\left(1+\frac{c}{n}\right)^{n}=e^{c}$, where $c$ is some (possibly matrix-valued) constant we obtain from equations $(9),(10)$

$$
\left[\begin{array}{l}
A_{2 N+1} \\
B_{2 N+1}
\end{array}\right]=\exp \left(-i k(a+b) \sigma_{3}\right) \exp \left(i\left(\left(k b+\frac{a q \xi}{2}\right) \sigma_{3}+\frac{i a q \eta}{2} \sigma_{2}\right)\right)\left[\begin{array}{c}
A_{0} \\
B_{0}
\end{array}\right]
$$

We denote the two coefficients in the second exponent as

$$
f=k b+a q \frac{\xi}{2}, \quad d=a q \frac{\eta}{2}
$$

making use of the relation

$$
\left(f \sigma_{3}+i d \sigma_{2}\right)^{2}=f^{2}-d^{2}=\phi^{2}
$$

we can expand the second exponent on the right hand side of Eq (11) in a Taylor series. After collecting corresponding terms we obtain

$$
e^{i\left(\left(k b+a q \frac{\xi}{2}\right) \sigma_{3}+i a q \frac{\eta}{2} \sigma_{2}\right)}=\cos \left(\sqrt{f^{2}-d^{2}}\right)+\frac{i\left(f \sigma_{3}+i d \sigma_{2}\right)}{\sqrt{f^{2}-d^{2}}} \sin \left(\sqrt{f^{2}-d^{2}}\right)
$$

Defining $z=k(a+b)$, we , therefore, obtain

$$
\left[\begin{array}{c}
A_{2 N+1} \\
B_{2 N+1}
\end{array}\right]=\left[\begin{array}{cc}
e^{-i z}\left(\cos \phi+i f \frac{\sin (\phi)}{\phi}\right) & i e^{-i z} d \frac{\sin (\phi)}{\phi} \\
-i e^{i z} d \frac{\sin (\phi)}{\phi} & e^{i z}\left(\cos \phi-i f \frac{\sin (\phi)}{\phi}\right)
\end{array}\right]\left[\begin{array}{l}
A_{0} \\
B_{0}
\end{array}\right]
$$

The corresponding expression for a single barrier of the same total width and location is [3]

$$
\left[\begin{array}{l}
A \\
B
\end{array}\right]=\left[\begin{array}{cc}
e^{-i z}\left(\cos (a q)+i \frac{\xi}{2} \sin (a q)\right) & i e^{-i z} \frac{\eta}{2} \sin (a q) \\
-i e^{i z \frac{\eta}{2} \sin (a q)} & e^{i z}\left(\cos (a q)-i \frac{\xi}{2} \sin (a q)\right)
\end{array}\right]\left[\begin{array}{l}
A_{0} \\
B_{0}
\end{array}\right]
$$

One sees that the internal structure of the multiple barrier, in the limit of $N \rightarrow \infty$, is different (they coincide only if $b=0$ ). The determinant of the matrix on the right hand side of Eq (15) is unity.

As we have seen, the equations (9)-(15) were concerned with expressing the amplitudes of the transmitted and reflected parts $A_{2 N+1}, B_{2 N+1}$ of the wave function at the right hand side of the bounded system (at the point $\left.\frac{(a+b)}{2}\right)$ as functions of $A_{0}$, and $B_{0}$ at the left hand side of this system (at the point $-\frac{(a+b)}{2}$ ). We can find these amplitudes at an arbitrary point $-\frac{a+b}{2}<x<\frac{a+b}{2}$ by using the property of the system that it is an infinite sequence of potential barriers bounded at two sides, so the point $x$ is associated with some barrier $n$ and may be written as $x= \pm n\left(\frac{a}{N}+\frac{b}{N-1}\right)= \pm n p$, where $p=\frac{a}{N}+\frac{b}{N-1}$, and $n$ is in the range $1 \leq n \leq \frac{N}{2}$. The potential cycle $p$ can be expressed in terms of the total length $L$ as $p=\frac{L}{N}$, so $x= \pm n \frac{L}{N}$, or $n= \pm \frac{x N}{L}$. Since $x$ and $L$ are finite numbers $n$ must be infinite if $N$ is. Thus, the amplitudes of the transmitted and reflected parts $A_{2 n+1}, B_{2 n+1}$ of the wave function at the $n$-th potential barrier can be written as (compare with Eq (11)) 


$$
\begin{aligned}
& {\left[\begin{array}{l}
A_{2 n+1} \\
B_{2 n+1}
\end{array}\right]=\left[\begin{array}{cc}
e^{-i k(a+b)} & 0 \\
0 & e^{i k(a+b)}
\end{array}\right]\left(1+\frac{i}{N}\left(\left(k b+a q \frac{\xi}{2}\right) \sigma_{3}+i a q \frac{\eta}{2} \sigma_{2}\right)\right)^{n}\left[\begin{array}{c}
A_{0} \\
B_{0}
\end{array}\right]=} \\
& =\exp \left(-i k(a+b) \sigma_{3}\right)\left(1+\frac{i}{N}\left(\left(k b+a q \frac{\xi}{2}\right) \sigma_{3}+i a q \frac{\eta}{2} \sigma_{2}\right)\right)^{ \pm \frac{x N}{L}}\left[\begin{array}{c}
A_{0} \\
B_{0}
\end{array}\right]= \\
& =\exp \left(-i k(a+b) \sigma_{3}\right) \exp \left( \pm \frac{i x}{L}\left(f \sigma_{3}+i d \sigma_{2}\right)\right)\left[\begin{array}{c}
A_{0} \\
B_{0}
\end{array}\right]
\end{aligned}
$$

We have used Eqs (12) and the relation $\lim _{n \rightarrow \infty}\left(1+\frac{c_{1}}{n}\right)^{n c_{2}}=e^{c_{1} c_{2}}$, where $c_{1}$ and $c_{2}$ are arbitrary finite (possibly matrix-valued) constants. Now if we define $f_{1}=\frac{f x}{L}, d_{1}=\frac{d x}{L}$ we obtain $f_{1}^{2}-d_{1}^{2}=\frac{x^{2}}{L^{2}} \phi^{2}=\phi_{1}^{2}$, where $\phi$ is given by Eq (13). Thus, we may use all the equations written before (for the right hand side of the dense system at the point $x=\frac{a+b}{2}$ ) also for an arbitrary point $-\frac{a+b}{2}<x<\frac{a+b}{2}$. This result provides a closed form for the wave function in the potential region which we shall discuss further in a succeeding publication.

Now, defining $e^{i \kappa}=\frac{\left(\cos (\phi)+i f \frac{\sin (\phi)}{\phi}\right)}{\sqrt{\left(\cos ^{2}(\phi)+f^{2} \frac{\sin ^{2}(\phi)}{\phi^{2}}\right)}}$, we can find from Eq (15) the transmission probability at the point $x=\frac{a+b}{2}$ by noting that at this point we have zero reflection, so $B_{2 N+1}=0$. Thus, we obtain for this probability

$$
\left|\frac{A_{2 N+1}}{A_{0}}\right|^{2}=\left|\frac{1}{e^{i z}\left(\cos (\phi)-i f \frac{\sin (\phi)}{\phi}\right)}\right|^{2}=\left|\frac{e^{i(\kappa-z)}}{\sqrt{\cos ^{2}(\phi)+f^{2} \frac{\sin ^{2}(\phi)}{\phi^{2}}}}\right|^{2}=\frac{1}{1+\frac{d^{2} \sin ^{2}(\phi)}{\phi^{2}}},
$$

where we have used Eqs (12). We see that the transmission probability reduces, when $b=0$, to the known transmisson probability of the one potential barrier system which is located at the same place and exposed to the same wave function as the infinite potential barrier system $[3,4]$,

$$
\left|\frac{A}{A_{0}}\right|^{2}=\frac{1}{\cos ^{2}(a q)+\frac{\xi^{2} \sin ^{2}(a q)}{4}} .
$$

We will see that the presence of a finite $b$ in Eq (18) results in a new possibility for the transmisson probability to reach unity without having to increase the energy as in the one potential barrier system. Clearly, if $a \rightarrow 0$ (no potential barrier), the transmission coefficient goes trivially to 1 . Figure 6 shows a three dimensional graph of the transmission probability from Eq (18) as a function of the energy $e$ and $c$. The total length is $L=70, v=60$, the range of $e$ is $61 \leq e \leq 120$, and that of $c$ is $0.01 \leq c \leq 5$. As expected the transmission probability tends to unity when the energy $e$ grows, but as seen from the graph it tends faster, even critically, to the neighbourhood of unity (even for small $e$ ) as $c$ increases through relatively small values (i.e., this effect is not simply due to $a \rightarrow 0$ ), that is, as the total spacing becomes larger (see Eq (2)). This is in agreement with the results obtained in the previous section for the $e>v$ case (see figures 2-3).

\section{SCATTERING CROSS SECTION}

We now study the scattering cross section of the bounded potential barrier system. For this we use the $S$ matrix which connects the outgoing waves $A_{2 N+1}$, and $B_{0}$ at the two sides of the system to the ingoing ones $B_{2 N+1}$ and $A_{0}$. That is, 


$$
\left[\begin{array}{c}
A_{2 N+1} \\
B_{0}
\end{array}\right]=\left[\begin{array}{ll}
S_{11} & S_{12} \\
S_{21} & S_{22}
\end{array}\right]\left[\begin{array}{c}
A_{0} \\
B_{2 N+1}
\end{array}\right]
$$

Using the last equation together with equation (15) (we denote the two dimensional matrix from $\mathrm{Eq}(15)$ by $Q$ where $\operatorname{det} Q=1)$ one obtains for the four components of the matrix $S$ $[3]$

$$
S_{11}=Q_{11}-\frac{Q_{12} Q_{21}}{Q_{22}}=\frac{1}{Q_{22}}, \quad S_{12}=\frac{Q_{12}}{Q_{22}}, \quad S_{21}=-\frac{Q_{21}}{Q_{22}}, \quad S_{22}=\frac{1}{Q_{22}}
$$

Now, in order to find the phase shifts we have to find the eigenvalues $\lambda_{ \pm}$from the following equation $\operatorname{det}(S-\lambda I)=\operatorname{det}\left[\begin{array}{cc}S_{11}-\lambda & S_{12} \\ S_{21} & S_{22}-\lambda\end{array}\right]=0$. That is,

$$
\begin{aligned}
& \lambda_{ \pm}=\frac{1}{Q_{22}}\left(1 \pm i \sqrt{\left|Q_{12}\right|^{2}}\right)=\frac{1 \pm \frac{i d \sin (\phi)}{\phi}}{e^{i z}\left(\cos (\phi)+i f \frac{\sin (\phi)}{\phi}\right)}=\frac{\left(1 \pm \frac{i d \sin (\phi)}{\phi}\right) e^{-i z}\left(\cos (\phi)-\frac{i f \sin (\phi)}{\phi}\right)}{\cos ^{2}(\phi)+\frac{f^{2} \sin ^{2}(\phi)}{\phi^{2}}}= \\
& =\frac{\cos (z) \cos (\phi)+\frac{f \sin (z) \sin (\phi)}{\phi} \pm d\left(\frac{\sin (z) \sin (\phi) \cos (\phi)}{\phi}-\frac{f \cos (z) \sin ^{2}(\phi)}{\phi^{2}}\right)}{\cos ^{2}(\phi)+\frac{f^{2} \sin ^{2}(\phi)}{\phi^{2}}}+ \\
& +\frac{i\left( \pm d\left(\frac{\sin (\phi) \cos (z) \cos (\phi)}{\phi}+\frac{f \sin (z) \sin ^{2}(\phi)}{\phi^{2}}\right)-\sin (z) \cos (\phi)+\frac{f \cos (z) \sin (\phi)}{\phi}\right)}{\cos ^{2}(\phi)+\frac{f^{2} \sin ^{2}(\phi)}{\phi^{2}}}
\end{aligned}
$$

According to the conventional phase shift theory $[3,4] \lambda_{ \pm}=e^{2 i \delta_{ \pm}}$, where $\delta_{ \pm}$are the phase shifts that correspond to the eigenvalues $\lambda_{ \pm}$. From the last relations we obtain

$$
\begin{aligned}
\cos \left(2 \delta_{ \pm}\right) & =\frac{\cos (z) \cos (\phi)+\frac{f \sin (z) \sin (\phi)}{\phi} \pm d\left(\frac{\sin (z) \sin (\phi) \cos (\phi)}{\phi}-\frac{f \cos (z) \sin ^{2}(\phi)}{\phi^{2}}\right)}{\cos ^{2}(\phi)+\frac{f^{2} \sin ^{2}(\phi)}{\phi^{2}}} \\
\sin \left(2 \delta_{ \pm}\right) & =\frac{ \pm d\left(\frac{\sin (\phi) \cos (z) \cos (\phi)}{\phi}+\frac{f \sin (z) \sin ^{2}(\phi)}{\phi^{2}}\right)-\sin (z) \cos (\phi)+\frac{f \cos (z) \sin (\phi)}{\phi}}{\cos ^{2}(\phi)+\frac{f^{2} \sin ^{2}(\phi)}{\phi^{2}}}
\end{aligned}
$$

The scattering amplitude is given by $S-1=e^{2 i \delta_{ \pm}}-1=2 i e^{i \delta_{ \pm}} \sin \left(\delta_{ \pm}\right)=2 \pi i T_{ \pm}$, and the cross section $\sigma_{ \pm}$is then obtained as

$$
\sigma_{ \pm}=4 \pi^{2}\left|T_{ \pm}\right|^{2}=4 \sin ^{2}\left(\delta_{ \pm}\right)=2\left(1-\cos \left(2 \delta_{ \pm}\right)\right)
$$

We see that $\sigma_{ \pm} \rightarrow 0$ for $e \rightarrow \infty$, since $d \approx O\left(\frac{1}{\sqrt{e}}\right) \rightarrow 0$ and $\frac{f}{\phi} \rightarrow 1$. It is further clear that for large $e$ the period of oscillation grows; the oscillations with respect to $e$ go as $\sqrt{e}$, and the period, for which $\sqrt{e+\Delta}=\sqrt{e}+2 \pi$, is determined by $\Delta=4 \pi^{2}+4 \pi \sqrt{e}$, therefore grows. Figure 7 shows a graph of $\sigma_{+}$as a function of the energy $e$ (the same graph is obtained also for $\left.\sigma_{-}\right)$. The total length $L$ is 70 , and $a=40$, so $b=30$. The potential $v$ is taken to be 70 , and the range of $e$ is $71 \leq e \leq 1000$. One sees the increase in period on this graph, but the decrease in amplitude would not become visible until $e>a^{2} v^{2} / 4$, i.e; for our case $e>2 \cdot 10^{6}$. An interesting property of $\sigma_{ \pm}$emerges when we relax the constraint of constant 
$L=a+b$. In this case we find that the dependence of the cross section upon $b$ is different from that upon $a$. That is, for the same value of $a$ the cross sections $\sigma_{ \pm}$, as functions of the energy $e$, depend also on $b$ only in a finite specific range that depends upon the value of $a$. For example, for $v=70$ and $a=10$, the cross section $\sigma_{+}$changes with $b$ in the range of $0 \leq b \leq 3.5 a$, and for $b>3.5 a$ the change in $\sigma_{+}$is so small that, as a function of $e$, can be considered constant. The same thing can be said also for $\sigma_{-}$. We find that the cross sections depend on the total width $a$ (for $b$ fixed) in such a manner that the periods of $\sigma_{ \pm}$ are inversely proportional to $a$. That is, as the total width $a$ of the potential barrier system grows the growth rate (with $e$ ) of the period of $\sigma_{ \pm}$becomes smaller.

We discuss now the energy level statistics [5] of the bounded dense array. To study this problem we use the $S$-matrix and the boundary value conditions at two remote boundaries of the system. That is, using periodic boundary conditions at the points $|x|=C$, where $C$ is much larger than the size $L=a+b$ of the system, we obtain $A_{2 N+1} e^{i k C}=A_{0} e^{-i k C}, \quad B_{2 N+1} e^{-i k C}=B_{0} e^{i k C}$. Thus, using the last two relations, and expressing the components of $S$ in terms of those of $Q$ (see Eq (21)), we write Eq (20) as

$$
\left[\begin{array}{c}
A_{2 N+1} \\
B_{0}
\end{array}\right]=e^{2 i k C}\left[\begin{array}{ll}
S_{11} & S_{12} \\
S_{21} & S_{22}
\end{array}\right]\left[\begin{array}{c}
A_{2 N+1} \\
B_{0}
\end{array}\right]=\frac{e^{2 i k C}}{Q_{22}}\left[\begin{array}{cc}
1 & Q_{12} \\
-Q_{21} & 1
\end{array}\right]\left[\begin{array}{c}
A_{2 N+1} \\
B_{0}
\end{array}\right]
$$

To obtain a non trivial solution for the vector $\left[\begin{array}{c}A_{2 N+1} \\ B_{0}\end{array}\right]$ we have to solve the following equation; det $\left[\begin{array}{cc}\frac{e^{2 i k C}}{Q_{22}}-1 & \frac{e^{2 i k C} Q_{12}}{Q_{22}} \\ -\frac{e^{2 i k} Q_{21}}{Q_{22}} & \frac{e^{2 i k C} C}{Q_{22}}-1\end{array}\right]=0$. The last equations, after substituting for the $Q$ 's from Eq (15), becomes

$$
\begin{aligned}
& \operatorname{det}\left[\begin{array}{cc}
\frac{e^{2 i k C}}{Q_{22}}-1 & \frac{e^{2 i k C} Q_{12}}{Q_{22}} \\
-\frac{e^{2 i k C} Q_{21}}{Q_{22}} & \frac{e^{2 i k C}}{Q_{22}}-1
\end{array}\right]=\frac{e^{4 i k C}}{Q_{22}^{2}}-\frac{2 e^{2 i k C}}{Q_{22}}+1+\frac{Q_{12} Q_{21} e^{4 i k C}}{Q_{22}^{2}}= \\
& =\cos (4 k C)\left(1+\frac{d^{2} \sin ^{2}(\phi)}{\phi^{2}}\right)+\cos (2 z)\left(\cos ^{2}(\phi)-\frac{f^{2} \sin ^{2}(\phi)}{\phi^{2}}\right)+\frac{2 f \sin (\phi)}{\phi}(\sin (2 z) \cos (\phi)- \\
& -\sin (2 k C+z))-2 \cos (2 k C+z) \cos (\phi)+i\left(\sin (4 k C)\left(1+\frac{d^{2} \sin ^{2}(\phi)}{\phi^{2}}\right)+\sin (2 z)\left(\cos ^{2}(\phi)-\right.\right. \\
& \left.\left.-\frac{f^{2} \sin ^{2}(\phi)}{\phi^{2}}\right)+\frac{2 f \sin (\phi)}{\phi}(\cos (2 k C+z)-\cos (2 z) \cos (\phi))-2 \sin (2 k C+z) \cos (\phi)\right)=0
\end{aligned}
$$

In order to obtain the spectrum we solve, numerically, the last equation for the energies that satisfy both its real and imaginary parts. Obtaining these energies we use the unfolding procedure [5] to transform to the more appropriate energies from which we may obtain energy level distribution. We find that the distribution of the energy level spacings depends sensitively upon the value of $c$. For small $c$ (small total interval $b$ and large total width $a$ ) the relevant distribution is more of the Poisson distribution type [5] than of the chaotic Wigner one, whereas when $c$ increases the corresponding distribution is more of the Wigner type than of the Poisson one. Figure 8 shows a histogram form of the level spacings distribution [5] of 102 energy levels obtained numerically for both cases of $e>v$ and $v>e$. The potential height is here taken to be $v=120$, and $C=90, L=20 c=19$. The continuous curve is the chaotic Wigner distribution [5] as obtained from a random matrix model [9], and the dashed 
one is the Poisson distribution [5]. One can see that the histogram-form curve resembles the chaotic Wigner one. The strong peak at 0.5 appears not consistent with the Poisson (dashed) curve. We remark that including levels only for $e>v$ results in a distribution which is not clearly of Wigner type; if we select only $v>e$ the distribution appears more clearly of Wigner type. We note that when we use Eq (27) for the $v>e$ case, the $f, d$ and $\phi$ that must be substituted in this equation are not those defined by equations (12),(13), but those defined in the following section in Eq (34).

In order to investigate further the properties of the bounded dense system we have passed a Gaussian wave packet through it and study its behaviour in the bounded potential region. We have used the complex packet

$$
\phi\left(x, t, x_{0}, p_{0}, w_{0}\right)=\frac{\sqrt{w_{0}} \pi^{\frac{1}{4}} e^{-\frac{p_{0}^{2}}{4 w_{0}^{2}} e^{\frac{w_{0}^{2}\left(i\left(x_{0}-x\right)-\frac{p_{0}}{2 w_{0}^{2}}\right)^{2}}{1-2 i t w_{0}^{2}}}}}{\sqrt{1-2 i t w_{0}^{2}}},
$$

where $x_{0}$ is the initial mean position of the packet in coordinate space, and $p_{0}$ and $w_{0}$ are the initial mean momentum, and initial width (uncertainty) of the momentum respectively in $p$ space. For our numerical simulations we have discretized space and time with a resolution of $d x=\frac{1}{7}$ and $d t=\frac{1}{50}$. This resolution ensures the condition $d t<d x^{2}$ which is necessary for a stable and steady performance of the numerical method used here [10]. For the other parameters we choose $m=\frac{1}{2}, w_{0}=\frac{1}{2}, x_{0}=-10, c=2.333 v=2$, and $p_{0}=3$. The last two chosen values ensure the condition of $e>v$. The dense system is arrayed between the points $x=-10$, and $x=10$. The units we are using for length and time are therefore; $x=\frac{x_{c m}}{\hbar}$ and $t=\frac{t_{s e c}}{m \hbar}$ (we take $p$ to be momentum in units $[m v]$ and $w_{0}$ the dispersion in $p)$. With this scale, we see that velocities in $\frac{\mathrm{cm}}{\mathrm{sec}}$ are related to our parametric velocities by $\frac{\Delta x_{c m}}{\Delta t_{s e c}}=\frac{1}{m} \frac{\Delta x_{c m}}{\Delta t_{s e c}}$. During and after the passage of the wave packet through the potential region its initial Gaussian form is strongly deformed. The point-type curve in figure 9 shows the form of the density of the wave packet which evolves from Eq (28) when the number of potential barriers is $N=4$, and the continuous graph is the form of this density for $N=150$. The potential barriers arrayed between $x=-10$ and $x=+10$ are not shown. Both curves in figure 9 are for the same time of $t=5.8$. Comparing these curves we see that for large $N$ the wave packet expands across the whole potential region more rapidly than the expansion for $N=4$. It has been suggested by Zaslavsky [2] (see also discussion in Ref [1]) that this behaviour is characteristic of a system with classical Hamiltonian of chaotic type. Moreover, we see that the structure of the wave function for $N=150$ is of a much higher degree of complexity. For larger values of $t$ (not shown) one sees that the forms of the transmitted and reflected waves are also of higher complexity. These results depend sensitively on the value of $c$. The effect is most pronounced when $c$ is in the neighbourhood of 4 , whereas, as $c$ grows this effect diminishes until it completely disappears for very large $c$. Moreover, we obtain a significant transmission of the wave packet through the potential region even for the case of $v>e$, and the part of the wave packet that passes through this region increases as the number $N$ or $c$ or both of them grow. Note that we have found that the transmission of a plane wave grows rapidly with $c$, whereas it may be poorly transmitted when $c$ is very small; in accordance with the wave packet behavior just described.

We now study the problem of resonances associated with the dense system. In order to find them we find the resonances of the cross section $\sigma_{ \pm}$(see Eq (25)). Using Eqs (12),(13) 
and $\mathrm{Eq}(23)$ (or $\mathrm{Eq}(24)$ ) we find that these are found at the values of the energies $e$ that satisfy the following equation

$$
1+\frac{d^{2} \sin ^{2}(\phi)}{\phi^{2}}=0
$$

Eliminating the total interval $b$ (see Eq (2)) from the following equations and substituting in Eq (29) for $d$ and $\phi$ from Eqs (12)-(13), and also for $q$ and $k$ we obtain

$$
\begin{aligned}
& \sin \left(\sqrt{\frac{(a+a c+c L)}{(1+c)^{2}}(e(a+a c+c L)-a v(1+c))}\right)= \\
& = \pm \frac{2 i}{a v(1+c)} \sqrt{e(a+a c+c L)(e(a+a c+c L)-v a(1+c))}
\end{aligned}
$$

The last equation can be, of course, valid only if the energy $e$ is complex. We denote this energy as $e=e_{1}+i e_{2}$. The components $e_{1}$ and $e_{2}$ are found (see Appendix A) by solving the following two simultaneous equations.

$$
\begin{aligned}
& \sin \left(r_{1}^{\frac{1}{2}} \cos \left(\frac{\phi_{1}+2 \pi k}{2}\right)\right) \cosh \left(r_{1}^{\frac{1}{2}} \sin \left(\frac{\phi_{1}+2 \pi k}{2}\right)\right)= \pm r_{2}^{\frac{1}{2}} \cos \left(\frac{\phi_{2}+2 \pi k}{2}\right) \\
& \cos \left(r_{1}^{\frac{1}{2}} \cos \left(\frac{\phi_{1}+2 \pi k}{2}\right)\right) \sinh \left(r_{1}^{\frac{1}{2}} \sin \left(\frac{\phi_{1}+2 \pi k}{2}\right)\right)= \pm r_{2}^{\frac{1}{2}} \sin \left(\frac{\phi_{2}+2 \pi k}{2}\right),
\end{aligned}
$$

where $k=0,1$ and $r_{1}, \cos \left(\phi_{1}\right), \sin \left(\phi_{1}\right), r_{2}, \cos \left(\phi_{2}\right)$, and $\sin \left(\phi_{2}\right)$ are given respectively by Eqs (A3)-(A8) in Appendix A. We find numerically that there is no solution to equations $(31),(32)$ for very large values of $c$. The allowed range of $c$, for which these equations may be satisfied, depends upon the value of the total length of the system $L$; as $L$ increases the allowed range of $c$ expands. For all other values of $c$ outside these ranges we find no pole that satisfy the simultaneous equations $(31),(32)$. We note that the poles are more frequent

at the middle sections of these ranges than at their ends. As noted the absolute values of the complex energy must be greater than $v$ since we deal here with the $|e|>v$ case. It can be shown (see Appendix B) that as long as these absolute values are not very much larger than the potential $v$ the Eqs $(31),(32)$ can be solved for a very large number of values of $e_{1}$ and $e_{2}$ (dependent upon the values of $L$ and $c$ ). But when $e_{1}$ or both $e_{1}$ and $e_{2}$ become very large these two equations have no solution for any value of $L$ and $c$.

\section{THE $V>E$ CASE}

We discuss, now, the $v>e$ case. The matrix equations (4),(5) may also be used for the $v>e$ case but the middle matrix $T$ at the right hand side of $\mathrm{Eq}(5)$ has to be written as

$$
\begin{aligned}
& T_{11}=\cosh \left(\frac{a q}{N}\right)+\frac{\grave{\xi}}{2} \sinh \left(\frac{a q}{N}\right), \quad T_{12}=\frac{\grave{\eta}}{2} \sinh \left(\frac{a q}{N}\right) \\
& T_{21}=-\frac{\grave{\eta}}{2} \sinh \left(\frac{a q}{N}\right), \quad T_{22}=\cosh \left(\frac{a q}{N}\right)-\frac{\grave{\xi}}{2} \sinh \left(\frac{a q}{N}\right)
\end{aligned}
$$


$k$ is $\sqrt{\frac{2 m e}{\hbar^{2}}}, q$ is $\sqrt{\frac{2 m(v-e)}{\hbar^{2}}}$, and $\grave{\xi}$ and $\grave{\eta}$ are given by $\grave{\xi}=-i \eta=\frac{q}{i k}+\frac{i k}{q}, \quad \grave{\eta}=-i \xi=\frac{q}{i k}-\frac{i k}{q}$, where $\eta$ and $\xi$ are from $\mathrm{Eq}(7)$. We can continue through the same steps as those of the $e>v$ case and find that the equivalent of the $f, d$, and $\phi$ from Eqs (12),(13) are

$$
\grave{f}=k b-\frac{a q \eta}{2}, \quad \grave{d}=\frac{a q \xi}{2}, \quad \grave{\phi}^{2}=\left(\grave{f} \sigma_{3}-i \grave{d} \sigma_{2}\right)^{2}=\grave{f}^{2}-\grave{d}^{2}
$$

and the corresponding equations to (14)-(15) are

$$
\begin{gathered}
e^{i\left(\left(k b-\frac{a q \eta}{2}\right) \sigma_{3}-\frac{i a q \xi}{2} \sigma_{2}\right)}=\cos \left(\sqrt{\grave{f}^{2}-\grave{d}^{2}}\right)+\frac{i\left(\grave{f} \sigma_{3}-i \grave{d} \sigma_{2}\right)}{\sqrt{\grave{f}^{2}-\grave{d}^{2}}} \sin \left(\sqrt{\grave{f}^{2}-\grave{d}^{2}}\right) \\
{\left[\begin{array}{l}
A_{2 N+1} \\
B_{2 N+1}
\end{array}\right]=\left[\begin{array}{cc}
e^{-i z\left(\cos \grave{\phi}+\frac{i \grave{f} \sin (\grave{\phi})}{\grave{\phi}}\right)} & -i e^{-i z \frac{\grave{d} \sin (\grave{\phi})}{\grave{\phi}}} \\
i e^{i z \frac{\grave{d} \sin (\grave{\phi})}{\grave{\phi}}} & e^{i z}\left(\cos \grave{\phi}-i \frac{\grave{f} \sin (\grave{\phi})}{\grave{\phi}}\right)
\end{array}\right]\left[\begin{array}{l}
A_{0} \\
B_{0}
\end{array}\right]}
\end{gathered}
$$

From the last two equations we can find the transmission probability for the $v>e$ case (in an analogous way to the $e>v$ case)

$$
\left.\left|\frac{A_{2 N+1}}{A_{0}}\right|^{2}=\mid \frac{1}{e^{i z}\left(\cos (\grave{\phi})-\frac{i f \sin (\grave{\phi})}{\grave{\phi}}\right.}\right)\left.\right|^{2}=\left|\frac{e^{i(\grave{\kappa}-z)}}{\sqrt{\cos ^{2}(\grave{\phi})+\frac{\grave{f}^{2}\left(\sin ^{2}(\grave{\phi})\right.}{\grave{\phi}^{2}}}}\right|^{2}=\frac{1}{1+\frac{\grave{d}^{2}\left(\sin ^{2}(\grave{\phi})\right.}{\grave{\phi}^{2}}}
$$

The $e^{i \kappa}$ is the same as the $e^{i \kappa}$ from the previous section (see the inline equation prior to Eq (18)) except that we substitute from Eq (34). Here, as for the $e>v$ case, the last expression reduces, when $b=0$, to the known transmission probability [3] for the one barrier located at the same place and exposed to the same wave function.

$$
\left|\frac{A}{A_{0}}\right|^{2}=\frac{1}{\cosh ^{2}(a q)+\frac{\eta^{2}\left(\sinh ^{2}(a q)\right)}{4}}
$$

As for the $e>v$ case the presence of a finite $b$ yields a new possibility for the transmission probability to be 1 even when $v \gg$. Figure 10 is a three dimensional surface of the transmission probability from Eq (37) as a function of the energy $e$ and $c$. The range of $e$ is $150 \leq e \leq 192$, and that of $c$ is $0.01 \leq c \leq 5$ which is the same range as in figure 6 . The potential $v$ is 200. As for the $e>v$ case the transmission probability tends to unity as $c$ increases.

We can continue, in a parallel way to the $e>v$ case, and find the scattering cross section from the corresponding $\grave{S}$-matrix which is found from Eq (36) (We denote the matrix in this equation by $\grave{Q})$ to be $\grave{S}=\frac{1}{\grave{Q}_{22}}\left[\begin{array}{cc}1 & \grave{Q}_{12} \\ \grave{Q}_{21} & 1\end{array}\right]$. From the Eq $\operatorname{det}(\grave{S}-\grave{\lambda} I)=0$ (see the equivalent discussion for the $e>v$ case) we can determine the corresponding eigenvalues $\grave{\lambda}_{ \pm}$which are the same as those found in Eq (22) except for the different $\grave{f}, \grave{d}$ and $\grave{\phi}$. From these $\grave{\lambda}_{ \pm}$we can write equations corresponding to $(23),(24)$, and from these equations the cross section $\grave{\sigma}_{ \pm}$may be obtained in an equivalent way to that of the $e>v$ case. It is found that also here the period of $\grave{\sigma}_{ \pm}$becomes larger as $e$ increases. As for the $e>v$ case, if we release the 
condition of a constant $L=a+b$, we find that the dependence of the cross sections $\grave{\sigma}_{ \pm}$, as functions of the energy $e$, upon the total interval $b$ is non-trivial only for a specific finite range which depends upon $a$. For example, for $v=140$, $e$ in the range $1 \leq e \leq 120$, and $a=10$ the cross section $\grave{\sigma}_{+}$, as a function of $e$, changes with $b$ only in the range $0<b \leq 8.7 a$. For any other value of $b>8.7 a$ we obtain $\grave{\sigma}_{+}=0$. The range of dependence upon $b$ becomes smaller as $a$ grows. for example, when $a$ grows from the former value of 10 to 15 the former range of $b$ becomes smaller by almost a factor of 3 , so that the new range in which $\grave{\sigma}_{+}$, as a function of $e$, changes with $b$ is $0<b \leq 3.13 a$. That is, for any other value of $b>3.13 a$ we obtain $\grave{\sigma}_{+}=0$.

We note that although the periods of $\bar{\sigma}_{ \pm}$become larger as the energy $e$ grows, the rate of growth is smaller compared to that of the $e>v$ case, and as for the later case, the total width $a$ and the rate of growth of the periods of $\grave{\sigma}_{ \pm}$are inversely proportional. That is, as $a$ grows the growth rate of these periods becomes small.

We discuss now the issue of resonances for the $v>e$ case. We may use, for that matter, the equation corresponding to Eq (29) of the $e>v$ case, except that we substitute the $\grave{f}, \grave{d}$ and $\dot{\phi}$ from $\mathrm{Eq}(34)$ and also the $q$ of the $v>e$ case. We obtain the following equation

$$
\begin{aligned}
& \sin \left(\sqrt{\frac{(a+a c+c L)}{(1+c)^{2}}(e(a+a c+c L)-a v(1+c))}\right)= \\
& = \pm \frac{2 i}{a v(1+c)} \sqrt{e(a+a c+c L)(e(a+a c+c L)-v a(1+c))}
\end{aligned}
$$

We now differentiate between two cases: The first is when $v a(1+c)<e(a+a c+c L)$ in which case the last equation is identical to Eq (30), and so we can use the two simultaneous equations (31),(32) in order to find the real and imaginary parts $e_{1}$ and $e_{2}$ of the energy $e$. The essential difference between $\mathrm{Eq}(30)$ and $\mathrm{Eq}(39)$ (when $v a(1+c)<e(a+a c+c L)$ ) is that in $\mathrm{Eq}(30)$ we have $e>v$, whereas here $v>e$. As for the $e>v$ case, the allowed ranges of $c$ depend upon the values of $L$ such that as $L$ increases these ranges grow.

We can prove, in an analogous manner to the $e>v$ case (see Appendix B), that the energies that may be considered as poles of the cross sections $\grave{\sigma}_{ \pm}$can not assume very large values (although here these energies have to satisfy the condition $v>e$ ).

The second case is when we have in Eq (39) $v a(1+c)>e(a+a c+c L)$, in which we obtain

$$
\begin{aligned}
& i \sinh \left(\sqrt{\frac{(a+a c+c L)}{(1+c)^{2}}(a v(1+c)-e(a+a c+c L))}\right)= \\
& =\mp \frac{2}{a v(1+c)} \sqrt{e(a+a c+c L)(v a(1+c)-e(a+a c+c L))}
\end{aligned}
$$

This equation can be solved only for complex energies $e=e_{1}+i e_{2}$. As in the $e>v$ case we eliminate the square roots and the complex character from both sides of $\mathrm{Eq}(40)$ by using the deMoivre theorem from Eq (A1) in Appendix A and the following hyperbolic sine addition formula [16] $\sinh (x+i y)=\sinh (x) \cos (y)+i \cosh (x) \sin (y)$. Thus, comparing separately the reals and imaginaries we obtain the following two simultaneous equations from which we try to find the real and imaginary parts of the energy 


$$
\begin{aligned}
& -\cosh \left(r_{1}^{\frac{1}{2}} \cos \left(\frac{\phi_{1}+2 \pi k}{2}\right)\right) \sin \left(r_{1}^{\frac{1}{2}} \sin \left(\frac{\phi_{1}+2 \pi k}{2}\right)\right)=\mp r_{2}^{\frac{1}{2}} \cos \left(\frac{\phi_{2}+2 \pi k}{2}\right) \\
& \sinh \left(r_{1}^{\frac{1}{2}} \cos \left(\frac{\phi_{1}+2 \pi k}{2}\right)\right) \cos \left(r_{1}^{\frac{1}{2}} \sin \left(\frac{\phi_{1}+2 \pi k}{2}\right)\right)=\mp r_{2}^{\frac{1}{2}} \sin \left(\frac{\phi_{2}+2 \pi k}{2}\right)
\end{aligned}
$$

where $k=0,1$, and $\grave{r}_{1}, \cos \left(\grave{\phi}_{1}\right), \sin \left(\grave{\phi}_{1}\right), \grave{r}_{2}, \cos \left(\grave{\phi}_{2}\right)$ and $\sin \left(\grave{\phi}_{2}\right)$ are given respectively by

$$
\begin{aligned}
& \grave{r}_{1}=r_{1}, \quad \sin \left(\grave{\phi}_{1}\right)=-\sin \left(\phi_{1}\right), \quad \cos \left(\grave{\phi}_{1}\right)=-\cos \left(\phi_{1}\right) \\
& \grave{r}_{2}=r_{2}, \quad \sin \left(\grave{\phi}_{2}\right)=\cos \left(\phi_{2}\right), \quad \cos \left(\grave{\phi}_{2}\right)=-\sin \left(\phi_{2}\right)
\end{aligned}
$$

The variables $r_{1}, \cos \left(\phi_{1}\right), \sin \left(\phi_{1}\right), r_{2}, \cos \left(\phi_{2}\right)$ and $\sin \left(\phi_{2}\right)$ are those of the $e>v$ case and are given by equations (A3)-(A8) in Appendix A. It has been turned out, numerically, that there is no solutions to the two simultaneous equations $(41),(42)$ that satisfy the condition of $v a(1+c)>e(a+a c+c L)$.

We note that when we have released the condition of constant $L$ we find (see the discussion on the cross-section $\grave{\sigma}_{ \pm}$before Eq (39)) that the cross sections $\grave{\sigma}_{ \pm}$become and remain zero for all values of $b$ that exceed some limiting value that depends upon the values of $a$. We have, also, found that these limiting values of $b$ become smaller as $a$ becomes larger. That is, in these cases the cross sections $\grave{\sigma}_{ \pm}$certainly have no poles. Now, when $a$ becomes large the probability that the difference $v a(1+c)-e(a+a c+c L)$ will be positive increases, and in this case, as we have just found, the cross sections $\grave{\sigma}_{ \pm}$have no poles in accordance with our discussion here.

Summarizing the subject of poles in the last two sections we see that for the $|e|>v$ case we find a very large number of different poles in large ranges of $c$, where the extent of these ranges depends upon the values of $L$. When $v>|e|$ the existence of poles depends upon the difference $v a(1+c)-|e|(a+a c+c l)$. That is, if this difference is negative then poles are found to the scattering cross sections $\grave{\sigma}_{ \pm}$, although in smaller ranges of $c$ compared to the corresponding ranges of the $|e|>v$ case. When the difference $v a(1+c)-|e|(a+a c+c L)$ is positive no pole of $\grave{\sigma}_{ \pm}$is found.

\section{CONCLUDING REMARKS}

We have discussed the properties of a large number $N$ of one-dimensional potential barriers arranged in a finite region of the $x$ axis. We use both a $4 N \times 4 N$ matrix method for finite $N$ and the transfer matrix method for the infinite array of these potential barriers along the finite region. We have discussed both cases of $e>v$ and $v>e$ and for both cases we found that the ratio of the total intervals between these potential barriers to their total width is an important parameter that determines the properties of the above mentioned variables. For example, when this ratio increases the transmission coefficient, for both cases of $e>v$ and $v>e$, of the passing plane wave or wave packet tends to the unity value even when the initial energies of these waves are very small. A similar effect was found [8] in a classical diffusion system with a high density of imperfect traps for which the survival probability [7] of classical particles passing through it tends to unity when the interval between the traps 
increases. Another system that was found $[13,14]$ to demonstrate the same behaviour is the array of identical optical analyzers, such as Nicol prism [15], so that when the number of them, along a finite interval, becomes very large a beam of light passes through them with the same initial polarization and intensity it had before the passage.

We have shown in this paper that a potential constructed of a large number of identical barriers can induce the type of behaviour as observed in the neighbourhood of tunneling barriers [1], interpreted in these references as chaotic-like. One may consider, as for the parallel drawn [1] between an unstable fixed point of the classical problem and quantum chaotic-like behaviour for the single barrier tunneling problem, a classical analog to the problem studied here. The repetitive potential in the bounded region, approached from above (for $e>v$ ), appears as an accumulation of unstable fixed points. The single wide barrier, on the other hand, is quasi-stable when approached from above; it is only the tunneling configuration in this case that has a strong analogy to the effect of a separatrix. For $c$ large, when the potential barriers are relatively well separated, we see an apparent chaotic-like effect most strongly through the Wigner type level distribution, and when $c$ is small the distribution moves toward Poisson type.

\section{ACKNOWLEDGEMENT}

We wish to thank D. Pearson and W. Amrein for discussions at an early stage of this work

\section{APPENDIX A}

Eqs (31)-(32) are obtained after eliminating the square roots and the complex nature from both sides of Eq (30). We do this by using the following two trigonometric relations [16].

$$
(r \cos (\phi)+i r \sin (\phi))^{\frac{1}{n}}=r^{\frac{1}{n}}\left(\cos \left(\frac{\phi+2 \pi k}{n}\right)+i \sin \left(\frac{\phi+2 \pi k}{n}\right)\right),
$$

where $n$ is any positive integer and $k=0,1,2 \ldots n-1$.

$$
\sin (a \pm i b)=\sin (a) \cos (i b) \pm \cos (a) \sin (i b)=\sin (a) \cosh (b) \pm i \cos (a) \sinh (b)
$$

Comparing separately the real and the imaginary parts of both sides we obtain the two simultaneous Eqs (31),(32) from which we can determine the components $e_{1}$ and $e_{2}$ of the energies $e$ that satisfy Eq (30). The six variables of Eqs (31),(32) that depends upon the cordinates $(r, \phi)$ are given by

$$
\begin{gathered}
r_{1}=\sqrt{\frac{(a+a c+c L)^{2}}{(1+c)^{4}}\left(\left(e_{1}(a+a c+c L)-v a(1+c)\right)^{2}+\left(e_{2}(a+a c+c L)\right)^{2}\right)} \\
\cos \left(\phi_{1}\right)=\frac{e_{1}(a+a c+c L)-a v(1+c)}{\sqrt{\left(e_{1}(a+a c+c L)-a v(1+c)\right)^{2}+\left(e_{2}(a+a c+c L)\right)^{2}}}
\end{gathered}
$$




$$
\sin \left(\phi_{1}\right)=\frac{e_{2}(a+a c+c L)}{\sqrt{\left(e_{1}(a+a c+c L)-a v(1+c)\right)^{2}+\left(e_{2}(a+a c+c L)\right)^{2}}}
$$

$$
\begin{aligned}
& r_{2}=\sqrt{\frac{16(a+a c+c L)^{2}}{a^{4} v^{4}(1+c)^{4}}\left(\left(e_{2} v a(1+c)-2 e_{1} e_{2}(a+a c+c L)\right)^{2}+\left((a+a c+c L)\left(e_{1}^{2}-e_{2}^{2}\right)-e_{1} v a(1+c)\right)^{2}\right)} \\
& \cos \left(\phi_{2}\right)=\frac{e_{2} v a(1+c)-2 e_{1} e_{2}(a+a c+c L)}{\sqrt{\left(e_{2} v a(1+c)-2 e_{1} e_{2}(a+a c+c L)\right)^{2}+\left((a+a c+c L)\left(e_{1}^{2}-e_{2}^{2}\right)-e_{1} v a(1+c)\right)^{2}}} \\
& \sin \left(\phi_{2}\right)=\frac{(a+a c+c L)\left(e_{1}^{2}-e_{2}^{2}\right)-e_{1} v a(1+c)}{\sqrt{\left(e_{2} v a(1+c)-2 e_{1} e_{2}(a+a c+c L)\right)^{2}+\left((a+a c+c L)\left(e_{1}^{2}-e_{2}^{2}\right)-e_{1} v a(1+c)\right)^{2}}}
\end{aligned}
$$

\section{APPENDIX B}

We show that there is no solution to Eqs (31)-(32) for very large values of $e_{1}$ or of both $e_{1}$ and $e_{2}$. In the first case we have $e_{1}>e_{2}$ and we obtain from equations (A3)-(A8) In Appendix A

$$
\begin{aligned}
& \sin \left(\phi_{1}\right) \approx \cos \left(\phi_{2}\right) \approx 0, \quad \cos \left(\phi_{1}\right) \approx \sin \left(\phi_{2}\right) \approx 1 \\
& r_{1} \approx \frac{e_{1}(a+a c+c L)^{2}}{(1+c)^{2}}, \quad r_{2} \approx \frac{4 e_{1}^{2}(a+a c+c L)^{2}}{a^{2} v^{2}(1+c)^{2}}
\end{aligned}
$$

Using these approximations we can write the two simultaneous equations (31),(32) for the $k=0$ as (we note that the following two equations do not change their forms if this $k$ assumes its second value of $k=1$ ).

$$
\begin{aligned}
& \sin \left(r_{1}^{\frac{1}{2}} \cos \left(\pi k_{1}\right)\right) \cosh \left(r_{1}^{\frac{1}{2}} \sin \left(\frac{\pi k_{1}}{2}\right)\right)= \pm r_{2}^{\frac{1}{2}} \cos \left(\frac{\pi}{4}+\frac{\pi k_{1}}{2}\right) \\
& \cos \left(r_{1}^{\frac{1}{2}} \cos \left(\pi k_{1}\right)\right) \sinh \left(r_{1}^{\frac{1}{2}} \sin \left(\frac{\pi k_{1}}{2}\right)\right)= \pm r_{2}^{\frac{1}{2}} \sin \left(\frac{\pi}{4}+\pi k_{1}\right)
\end{aligned}
$$

It can be seen that $\mathrm{Eq}(\mathrm{B} 3)$ is not satisfied for $k_{1}=0$ or an even $k_{1}$. We, now, show that these equations are not satisfied for any uneven $k_{1}$ either. For these $k_{1}$ these two equations can be written as

$$
\sin \left(r_{1}^{\frac{1}{2}}\right) \cosh \left(r_{1}^{\frac{1}{2}}\right)= \pm r_{2}^{\frac{1}{2}} \sin \left(\frac{\pi}{4}\right)
$$




$$
\cos \left(r_{1}^{\frac{1}{2}}\right) \sinh \left(r_{1}^{\frac{1}{2}}\right)= \pm r_{2}^{\frac{1}{2}} \cos \left(\frac{\pi}{4}\right)
$$

Squaring the two sides of both equations we realize that their right sides are the same. So equating the left sides we obtain

$$
\tan ^{2}\left(r_{1}^{\frac{1}{2}}\right)=\tanh ^{2}\left(r_{1}^{\frac{1}{2}}\right)
$$

In order for the last equation to be valid the variable $r_{1}^{\frac{1}{2}}$ must be small, but it is given that $e_{1}$ is very large, so $r_{1}^{\frac{1}{2}}$ must also be very large (see Eq (B1)) and the equation (B6) can not be satisfied. Thus, when $e_{1} \gg e_{2}$ we find no poles of the cross section from (29).

The same consequence is obtained also when both $e_{1}$ and $e_{2}$ are very large, so that we can write $e_{1} \approx e_{2}$. In this case we obtain

$$
\sin \left(\phi_{1}\right) \approx \cos \left(\phi_{1}\right) \approx \frac{1}{\sqrt{2}}, \quad \cos \left(\phi_{2}\right) \approx-1, \quad \sin \left(\phi_{2}\right) \approx 0
$$

and the corresponding two approximate simultaneous equations for this case are

$$
\begin{gathered}
\sin \left(r_{1}^{\frac{1}{2}} \cos \left(\frac{\pi}{8}+\frac{\pi k_{1}}{4}\right)\right) \cosh \left(r_{1}^{\frac{1}{2}} \sin \left(\frac{\pi}{8}+\frac{\pi k}{4}\right)\right)= \pm r_{2}^{\frac{1}{2}} \cos \left(\frac{\pi+2 \pi k_{1}}{2}\right) \\
\cos \left(r_{1}^{\frac{1}{2}} \cos \left(\frac{\pi}{8}+\frac{\pi k_{1}}{4}\right)\right) \sinh \left(r_{1}^{\frac{1}{2}} \sin \left(\frac{\pi}{8}+\frac{\pi k_{1}}{4}\right)\right)= \pm r_{2}^{\frac{1}{2}} \sin \left(\frac{\pi k_{1}}{2}\right)
\end{gathered}
$$

It can be seen that the first equation can not be solved for any $k_{1}$ (even or uneven). Thus, we see that very large energies can not be solutions of $(31),(32)$. 


\section{REFERENCES}

[1] A. K. Pattanayak and W. C. Schieve, Phys. Rev. Lett, 72, 2855, (1994); Y. Ashkenazy et al, Phys. Rev. Lett, 75, 1070, (1995)

[2] G. M. Zaslavsky, Phys. Rep, 80, 157 (1981)

[3] Quantum mechanics second edition by E.Merzbacher, John Wiley and sons, (1961)

[4] Quantum mechanics by C. C. Tannoudji, B. Diu, And Franck Laloe, John Wiley and Sons (1977)

[5] The transition to chaos in conservative classical systems: Quantum manifestations, L. E. Reichel, Springer, Berlin, 1992 ;E. Haller, H. Koppel and L. S. Cederbaum, Chem. Phys. Lett, 101, 215-220, (1983); T. A. Brody et al, Rev. Mod. Phys, 53, 385, (1985)

[6] A. M. Frishman and S. A. Gurvitz, Phys. Rev B, 47, 16348-16352, (1993)

[7] R. V. Smoluchowski, Z. Phys. Chem., Stoechiom. Verwandtschaftsl, 29, 129, (1917); Diffusion and reactions in fractals and disordered media by D. Ben-Avraham And S. Havlin, Cambridge, Camgridge University Press, 2000;

[8] D. Bar, Phys. Rev. E, 64, 02618, (2001).

[9] F. J. Dyson and M. L. Mehta, J. Math. Phys, 4, 489, (1963)

[10] Quantum Mechanics Using Maple, Horbatsch Marko, Springer, Berlin, (1995)

[11] K. W. Yu, Computers in Physics 4, 176-178, (1990)

[12] It can be shown that the matrix $T^{N}$ does not depend also on the number of potential barriers $N$. That is, its elements as given by the set (3) receive the same values for each positive integer $N$.

[13] A. Peres, Am. J. Phys, 48, 931, (1980).

[14] D. Bar and L. P. Horwitz, Int. J. Theor. Phys, 40, 10, 1697-1713, (2001).

[15] M. Born and E. Wolf, Principles of Optics, (Cambridge University Press, Cambridge, 1999).

[16] Mathematical Handbook, Murray. R. Spiegel, Schaum's outline series, McGraw-Hill Book Company, (1968) 


\section{FIGURES}

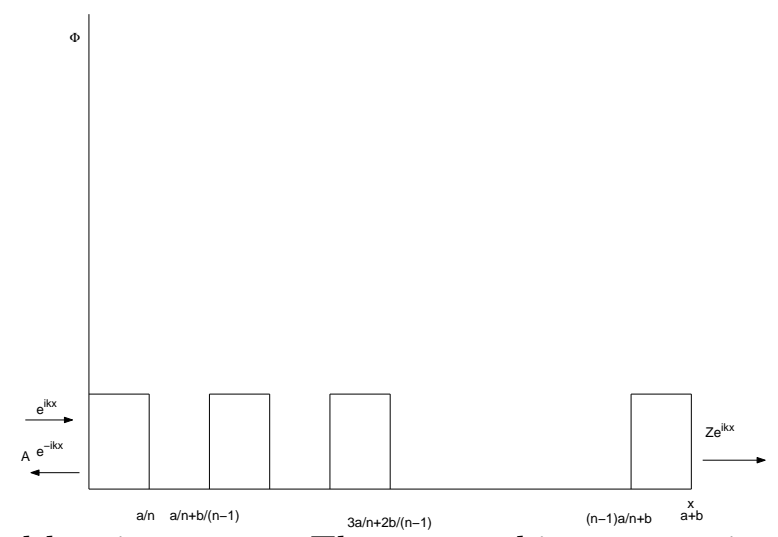

FIG. 1. The $n$ potential barrier system. The approaching, transmitted and reflected waves are shown at right and left.

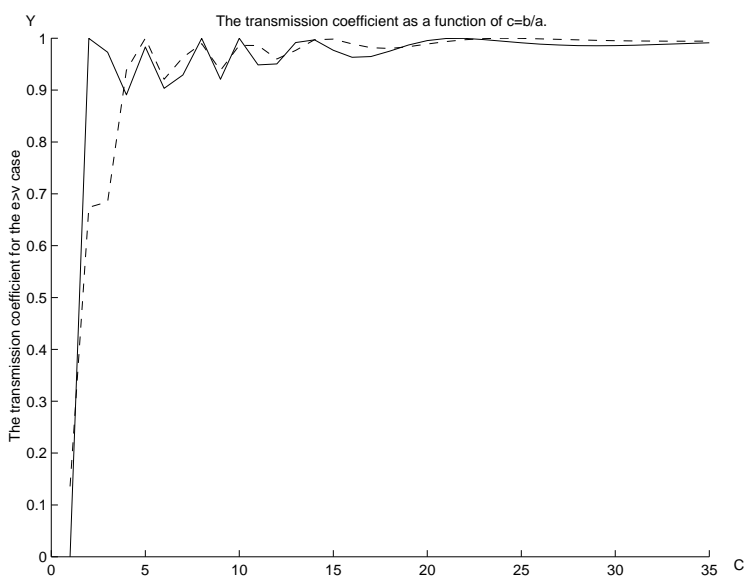

FIG. 2. The continuous curve is the plot of the transmission coefficient for $N=30$, and the dashed one is for $N=40$, both as functions of $c$, and for the $e>v$ case. Note that for the larger $N$ the transmission coefficient tends to unity for smaller values of c.

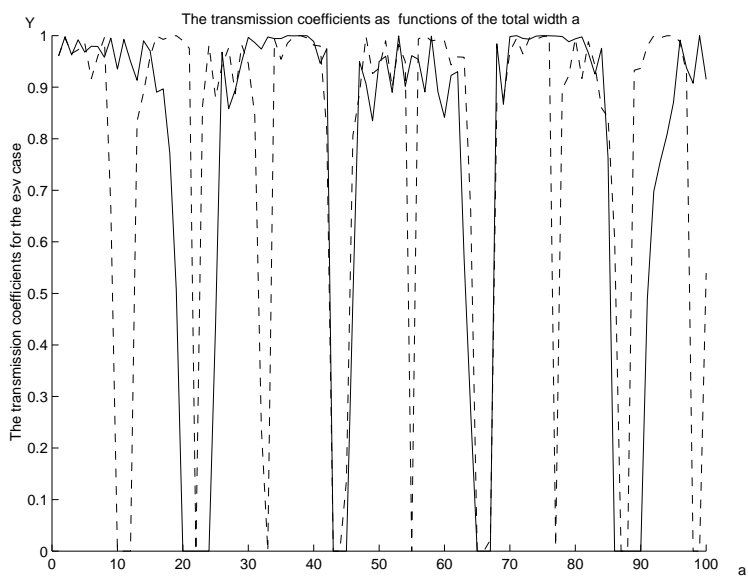

FIG. 3. The dashed curve is the plot of the transmission coefficient for $N=60$, and the continuous curve is for $N=120$, both as functions of $a$, and for the $e>v$ case. 




FIG. 4. The continuous curve is the plot of the transmission coefficient for $N=30$, and the dashed one for $N=50$, both as functions of $c$, and for the $v>e$ case. Note that as in figure 2 the transmission coefficient for the larger $N$ tends to unity at smaller values of $c$.

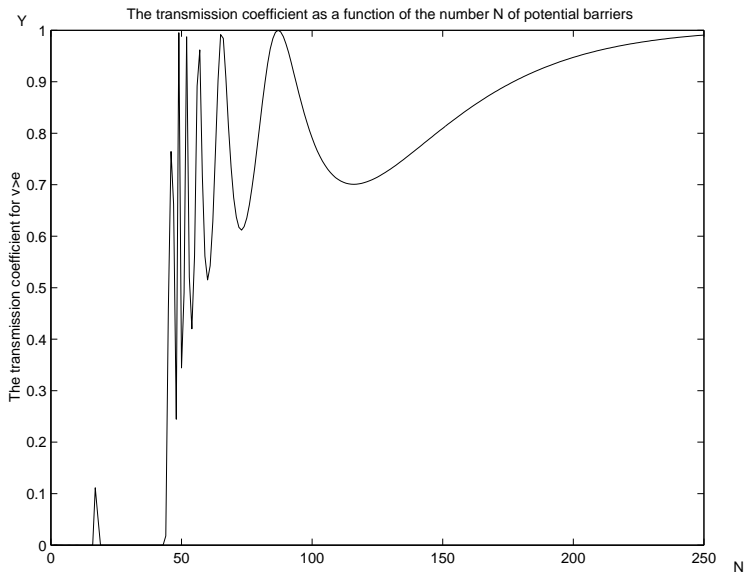

FIG. 5. The transmission coefficient as a function of $N$ for the $v>e$ case. The total width a is 8 , the potential $v$ is 202 , and the energy $e$ is 200 . Note that this coefficient tends to unity as $N$ increases.

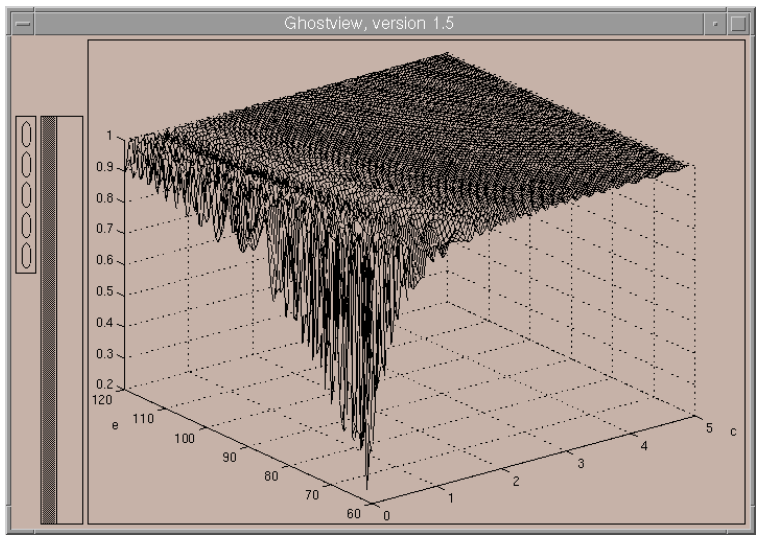


FIG. 6. A three dimensional graph of the transmission probability from Eq (18) for $e>v$ as a function of $c$ and the energy $e$. This graph is for a total system length of $L=70, v=60, c$ in the range $0.01 \leq c \leq 5$, and $61 \leq e \leq 120$. Note that the transmission probability as a function of $c$ tends to unity at a faster rate than as a function of $e$.



FIG. 7. The graph of the cross section $\sigma_{+}$from Eq (25) as a function of the energy $e$. This graph is for a total length of $L=70, a=40, v=70$, and for $e$ in the range $71 \leq e \leq 1000$. Note that the period of the graph becomes larger as the energy $e$ grows.

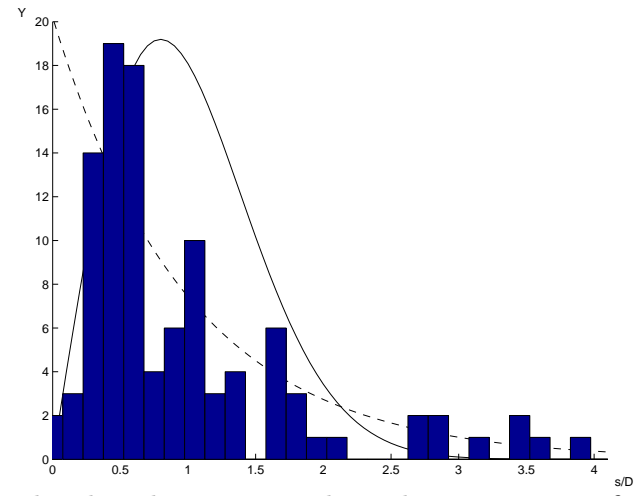

FIG. 8. The histogram is the level spacing distribution as a function of $s / D$ where $s$ is the spacing between neighbouring levels and $D$ is the mean spacing. The histogram is constructed from 102 energy levels found in the range $1 \leq e \leq 600, v$ is $120, C=90, L=20$, and $c=19$. The dashed curve is the Poisson distribution and the continuous graph is the Chaotic Wigner one. 


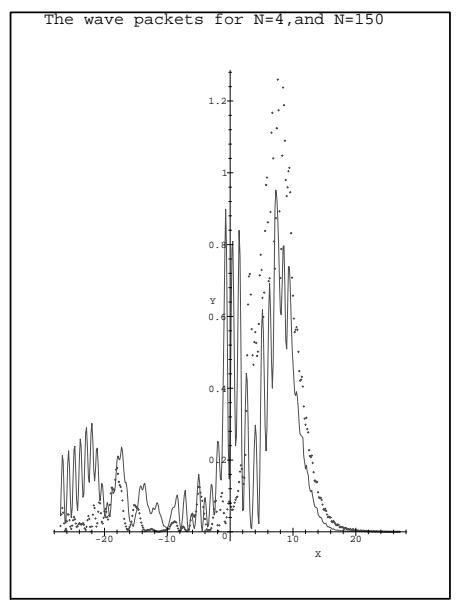

FIG. 9. The continuous curve is the form of the wave packet for $n=150$, and the point-type curve is the form for $n=4$. Both curves are at time $t=5.8$ in computer units.

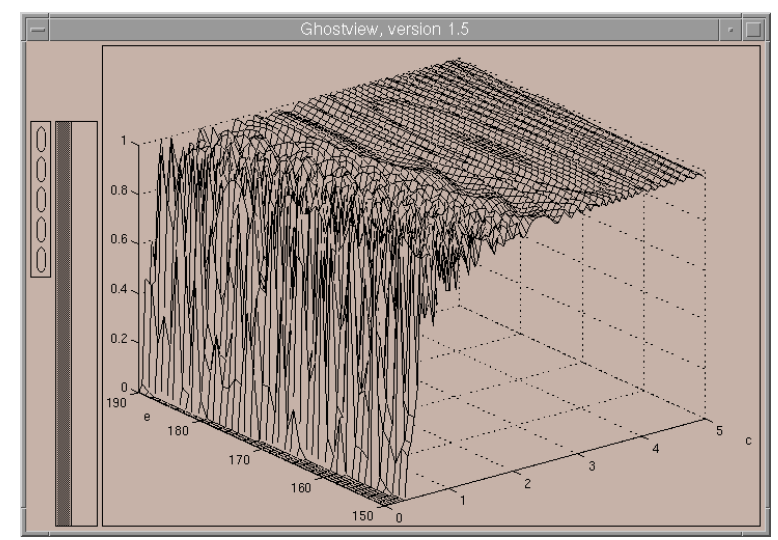

FIG. 10. A three dimensional graph of the transmission probability from Eq (37) for $v>e$ as a function of $c$ and the energy $e$. This graph is for $v=200, L=70, c$ in the range $0.01 \leq c \leq 5$, and $150 \leq e \leq 195$, We see from the figure that as the energy $c$ grows above the value 0.2 the transmission probability jumps to 1 . 